\title{
UNA UNIVERSIDAD AL SERVICIO DE LA INVESTIGACIÓN MULTIDISCIPLINARIA
}

\author{
THE UNIVERSITY IN THE RESEARCH SERVICE OF MULTIDISCIPLINARY \\ Raúl Alberto Arrarte Mera* \\ Docente Principal de la Facultad de Ciencias Contables
}

Universidad Nacional Mayor de San Marcos-UNMSM / Lima-Perú

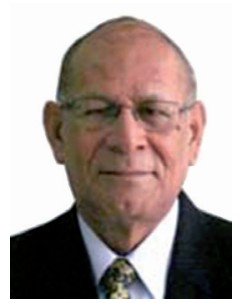

[Recepción: Setiembre de 2014/ Conformidad: Octubre 2014]

\section{RESUMEN}

Este trabajo es una propuesta de solución en el nuevo contexto de la Ley Universitaria No 30220, publicada el 09 de julio de 2014 para los futuros integrantes de la Asamblea Estatutaria de la Universidad Nacional Mayor de San Marcos, quienes serán elegidos por primera vez, en la historia de la vida universitaria por votación directa, secreta y universal por todos los estudiantes de pregrado y posgrado, así como, docentes y tendrán la delicada responsabilidad de redactar y aprobar su Estatuto. El método aplicado ha sido actualizar una tesis doctoral del suscrito aprobada el año 2005 denominada: "La Cooperación Universidad-Empresa-Estado en el Perú (Caso UNMSM 1997-2002)”, adaptada a las exigencias de la nueva Ley donde se establecen 16 capítulos, tres disposiciones complementarias transitorias, modificatorias y finales. Los objetivos son: Promover la investigación multidisciplinaria, la transparencia y la simplificación administrativa. A través de sus 11 organigramas estructurales, propone una reingeniería administrativa institucional, ahora formada por 20 facultades, integrada solamente a cinco (5) áreas por especialización: Ciencias Básicas; Ciencias de la Salud; Ingenierías; Económico-Empresariales y Humanidades. Una nueva estructura simplificada que facilitará, la eficacia, la eficiencia y la efectividad en la toma de decisiones económico-administrativas.

Palabras clave:

Asamblea estatutaria; investigación multidisciplinaria; reingeniería administrativa.

\begin{abstract}
This work is a proposal for settlement in the new context of the University Act No. 30220, published on July 9, 2014 for future members of the Statutory Assembly of the National University of San Marcos, who shall be elected for the first time in the story of university life by direct, secret and universal vote by all undergraduate and graduate students as well as teachers and have the delicate responsibility of drafting and approving its Statute. The method used has been updating a doctoral thesis subscribed approved 2005 entitled: "The University-Industry Cooperation-State in Peru (Case San Marcos 1997-2002)", adapted to the requirements of the new law which established 16 chapters three additional transitional provisions, amendments and final. The objectives are: To promote multidisciplinary research, transparency and administrative simplification. Through its 11 structural organization charts, administrative proposes an institutional reengineering, now consists of 20 faculties, comprising only five (5) areas of expertise: Basic Sciences; Health Sciences; engineering; Economic Business and Humanities. A new simplified structure that will facilitate the effectiveness, efficiency and effectiveness in making economic and administrative decisions.
\end{abstract}

\section{Keywords:}

Statutes Assembly; multidisciplinary investigation; administrative reengineering.

\footnotetext{
* Doctor en Ciencias Contables y Empresariales, Contador Público - UNMSM. Maestro en Administración - USMP.

Email: rarrartem@unmsm.edu.pe
} 


\section{INTRODUCCIÓN}

Este trabajo es una propuesta de solución para los futuros integrantes de la Asamblea Estatutaria de la Universidad Nacional Mayor de San Marcos (UNMSM), en el nuevo contexto de la Ley Universitaria No 30220, publicada en el diario (El Peruano: 2014), señala que serán elegidos por votación directa, secreta y universal por todos los estudiantes de pregrado y posgrado, así como, los docentes, quienes tendrán la delicada responsabilidad de redactar y aprobar su nuevo Estatuto, lo que resulta de singular importancia para el momento actual que vive el país.

Las universidades en el Perú se regían por la Ley No 23773 que fue promulgada el 9 de diciembre de 1983 bajo el gobierno constitucional del Arquitecto Fernando Belaúnde Terry, basada en la Constitución Política de 1979. Estaba dividida en 17 capítulos, 103 artículos, 17 disposiciones transitorias y dos disposiciones finales. El país salía de un largo periodo de dictadura militar (1968-1980). Los aspectos más significativos de esta ley fueron:

a) Cada universidad organiza y establece su régimen académico por facultades de acuerdo con sus características y necesidades (Art.9).

b) Las universidades pueden organizar institutos, escuelas, centros y otras unidades con fines de investigación, docencia y servicio.

c) El sistema electoral es de listas incompletas (Art.39).

Las deficiencias que nos dejó esta ley son las siguientes:

a) Disminución relativa de los aportes financieros del Estado al sostenimiento de la educación gratuita.

Esta situación ha fomentado que a mayores ingresos propios generados por la universidad, el gobierno de turno, reduce su participación. A este fenómeno se le llama el síndrome de Penélope, tal como le ocurría a la protagonista de La Odisea, respecto al regreso de su amado Ulises, Penélope tejía de día su chompa y de noche, la destejía. De la misma manera, ocurre con nuestras Universidades Públicas, a más eficiencia de nuestras universidades para autogenerar fondos propios, el Estado reduce su participación en esa mis- ma proporción, y por eso nuestras entidades nunca pueden avanzar en su proyección de investigaciones.

b) Restricciones normativas para modernizar la universidad pública.

La nueva ley en proceso de implementación contiene normativas más abiertas y flexibles; en especial, la relacionada con la disposición de los ingresos autogenerados. Así, el artículo $110.7^{\circ}$ establece que son recursos económicos, entre otros, "la prestación de servicios educativos de extensión, servicios de sus centros preuniversitarios, posgrado o cualquier otro servicio educativo distinto".

c) Una estructura organizacional compleja.

(Visitar el siguiente link: http://www.unmsm.edu.pe/ transparencia/archivos/organigrama_unmsm_2011.pdf)

Esta investigación de orden eminentemente técnico, hace una evaluación analítico-explicativa (Top-Down), de la cima a las bases de acuerdo al organigrama funcional de la UNMSM, para cuya finalidad la hemos dividido en las siguientes etapas:

1. Introducción.

2. El Statu Quo actual.

3. La reingeniería que necesita San Marcos.

4. Funciones de la Nueva Asamblea Estatutaria.

5. Conclusiones.

6. Bibliografía.

\section{EL STATU QUO ACTUAL}

\section{El Rectorado}

Tenía bajo su directo control, las siguientes oficinas:

a) Órgano de Control Institucional (OCI). La nueva disposición lo denomina "Comisión Permanente de Fiscalización” (Art. $77^{\circ}$ ) y reporta a la Asamblea Universitaria. Una rectificación muy saludable.

b) Radio TV San Marcos.

c) Oficina de Seguridad y Vigilancia.

d) Oficina de Comisiones Permanentes y Transitorias.

e) Centro de Informática.

f) Administración de la Red Telemática. 
g) Secretaría General.

h) Oficina General de Imagen Institucional.

i) Oficina General de Cooperación de Relaciones Interinstitucionales.

j) Oficina Central de Admisión.

k) Oficina General de Planificación.

1) Oficina General de Asesoría Legal.

m) Oficina Central de Calidad Académica y Acreditación.

n) Oficina Técnica del Estudiante (OTE).

o) Museo de Historia Natural.

p) Centro Cultural.

q)Instituto Raúl Porras Barrenechea.

r) Seminario de Historia Rural Andina.

s) Jardines Botánicos.

Como es de suponer, no es humanamente posible controlar cada una de estas oficinas. Por tanto, la planilla de asesores debió ser abultada.

En el nuevo organigrama que estamos proponiendo, (Ver ANEXOS Cuadro No 01), sólo quedarán bajo la administración directa del Rectorado, las oficinas marcadas en negrita seis (6) de diecinueve (19), las demás, serán asignadas a la Dirección de Administración y al Vicerrectorado Académico.

\section{Dirección General de Administración} (DIGA) cinas:

Tenía bajo su directo control, las siguientes ofi-

a) Oficina General de Servicios Generales, Operación y Mantenimiento.

b) Oficina General de Bienestar Universitario.

c) Oficina General de Economía.

d) Oficina General de Recursos Humanos.

e) Oficina General de Infraestructura Universitaria.

f) Clínica Universitaria.

g) Unidad de Fincas.

h) Oficina de Control Previo y Fiscalización.

En el nuevo organigrama que estamos proponiendo, (Ver ANEXOS Cuadro No 02), solo quedarán bajo la administración directa de la DIGA, las oficinas marcadas en negrita, además:

i) Se crea la Oficina General de Administración de Red Telemática que incluye: Oficina de Control Integral; Centro de Informática; Radio y TVy, Seguridad y Vigilancia donde se implementa en toda la Ciudad Universitaria cámaras de seguridad.

j) Se integra la Oficina de Bienestar Universitario, la Clínica Universitaria.

k) Se integra a la Oficina General de Infraestructura Universitaria: Unidad de Fincas.

\section{Vicerrectorado ACADÉmico (VRA)}

Tenía bajo su directo control, las siguientes oficinas:

a) Centro Preuniversitario.

b) Oficina General del Sistema de Bibliotecas y Biblioteca Central.

c) Sistema Único de Matrícula (SUM).

d) Centro de Producción Editorial e Imprenta.

e) Centro de Producción Librería y Distribución.

\section{f) Centro de Producción Fondo Editorial.}

En el nuevo organigrama que sugerimos, (Ver ANEXOS Cuadro $\mathrm{N}^{\circ} 03$ ), quedarán bajo la administración directa del VRA, las oficinas marcadas en negrita; es decir las mismas del régimen anterior, más:

g) Oficina Central de Admisión.

h) Oficina de Calidad Académica y Acreditación.

\section{VICE RECTORAdo de INVESTIGACIÓn (VRI)}

Tenía bajo su directo control, las siguientes oficinas:

a) Consejo Superior de Investigaciones (CSI).

b) Consejo de Transferencia e Innovación (CTI).

c) Consejo de Gestión de Investigación. (CGI)

Cuatro problemas aquejan a este sector:

1) Su estructura organizacional.

2) Su financiamiento.

3) La relación con el sector privado.

4) Bajas remuneraciones de los docentes. 


\section{ESTRUCTURA ORgANIZACIONAL DE LOS INSTI- TUTOS DE INVESTIGACIÓN}

La Universidad Nacional Mayor de San Marcos, (UNMSM) tiene 20 Facultades, las cuales tienen Unidades y/o Institutos de Investigación, pero por razones prácticas que da la experiencia cotidiana se ha dividido en cinco áreas:

ÁREA A: CIENCIAS BÁSICAS
Ciencias Biológicas
Ciencias Físicas
Ciencias Matemáticas
ÁREA B: CIENCIAS DE LA SALUD
Farmacia y Bioquímica.
Medicina
Medicina Veterinaria
Odontología
Psicología
ÁREA C: INGENIERÍAS
Ingeniería Electrónica y Eléctrica
Ingeniería Geológica, Minera, Metalúrgica y
Geográfica
Ingeniería Industrial
Ingeniería de Sistemas e Informática
Química e Ingeniería Química

ÁREA D: ECONÓMICO-EMPRESARIALES

Ciencias Administrativas

Ciencias Contables

Ciencias Económicas

ÁREA E: HUMANIDADES

Ciencias Sociales

Derecho y Ciencia Política

Educación

Letras y Ciencias Humanas

Según el organigrama de la UNMSM dependen del Vicerrectorado de Investigación:

1. Consejo Superior de Investigaciones.

2. Consejo de Transferencia e Innovación.

3. Consejo de Gestión de Investigación.

El centro de operaciones de esta área,es el Consejo Superior de Investigaciones, que promueve actualmente fondos concursales, en forma coordinada con los Institutos de Investigación de cada facultad.
Lo que se observa que los fondos no abastecen a la totalidad de profesores investigadores, la ley obliga a concursar, pasando por dos filtros: por un lado, el Instituto de Investigación y por otro, el Consejo de Facultad. La aprobación del proyecto termina politizándose, sin tener en cuenta la calidad del mismo, sino la importancia del cargo de quién lo presenta.

Pero esta estructura organizacional no promueve la investigación multidisciplinaria, sino unidisciplinaria, relacionado con la facultad a que corresponde. Los investigadores solo se conocen a nivel de facultad pero no de la universidad. Existe ausencia de integración.

La medida correctiva sería promover la investigación multidisciplinaria, teniendo como base a las Ciencias Empresariales, que actuarían como coordinadora y asesora de los diferentes grupos disciplinarios; logrando así, mejorar el rendimiento y mayor alcance de los proyectos. (Ver ANEXOS Cuadro No4, Estructura Organizacional VRI).

\section{Financiamiento de los proyectos de investigación}

La Ley Universitaria derogada recientemente, no permitía que los fondos autogenerados contribuyan a la financiación de los proyectos; sino más bien, obligaba a la inversión en infraestructura. La nueva Ley Universitaria sí toma en cuenta esta situación, pero omite las Asociaciones Público-Privadas, que podría ser materia de especificarlo en el futuro Reglamento de Investigaciones de la universidad.

\section{Relaciones con el sector privado}

Estamos convencidos que corresponde a la universidad desarrollar un puente de información hacia el sector privado, invitando a los representantes de entidades sectoriales, tales como: Sociedad Nacional de Industrias; Asociación de Exportadores; Cámara de Comercio de Lima; Sociedad Nacional de Minería; Sociedad Nacional de Pesquería, etc., y mostrar el potencial con que cuenta nuestra institución, para atender a la solución de sus problemas empresariales que pudieran generarse en el futuro, tanto en el desarrollo de nuevos productos, como en la modernización de los actuales. Nuestra institución debe llegar al empresariado con una oferta de servicios, la cual estamos en capacidad de realizar. Una especie 
de marketing que ahora mismo no desarrollamos, utilizando los medios tanto radiales como televisivos, buscando entrevistas diarias de nuestros especialistas en determinados programas que tienen que ver con la vida del sector empresarial, a la par de las universidades privadas.

Quizá una estrategia sería la especialización, cada universidad debería tener fortalezas específicas en determinado sector de la economía.

\section{Bajas remuneraciones docentes}

Según la Ley Universitaria, un docente desarrolla: actividades académicas, de investigación y de proyección social. La nueva Ley Universitaria contempla la categoría de Docente Investigador, con una carga lectiva de un curso por año y una bonificación especial de $50 \%$ adicional sobre sus haberes totales. Ingreso que alcanzaría a un promedio de $S / .9,000$ mensuales para la categoría de un Docente Principal a Tiempo Completo. Una medida muy plausible que el futuro del país, así lo exige.

En el nuevo organigrama que proponemos, (Ver ANEXOS Cuadro No 4), se plantea una renovación integral del VRI:

a) Consejo Directivo, en adelante el Consejo $\mathrm{Su}$ perior de Investigaciones, integrado por dos (2) representantes de cada área de la universidad: Ciencias Básicas; Ciencias de la Salud; Ingeniería; Económico-Empresariales y Humanidades.

b) Director que represente al Vicerrectorado.

c) Oficina de Coordinación Administrativa.

d) Oficina de Transferencia e Innovación.

e) Oficina de Cooperación Empresa-Universidad-Estado, que cumpliría la función de puente con los grupos de interés.

Paralela a esa decisión debería ir acompañada de la modificación al Reglamento Interno de Investigaciones que sólo permita la promoción de proyectos de investigación multidisciplinarios.

\section{CONSECUENCIAS QUE HA GENERADO ESTA ESTRUCTURA}

Las secuelas que ha generado esta estructura desde 1920 hasta 2014, son un desfase de más de 94 años, y son las siguientes:
1. Elefantiásica, irracional y burocrática.

2. Las universidades no tienen por qué tener la misma jerarquía, autonomía, y organización. La verdadera autonomía está en definir su propia estructura organizacional en función de sus necesidades y los objetivos establecidos por el Estado peruano.

3. Todos los modelos asumen el autofinanciamiento.

4. El desbalance de poder político entre el Rector y los Vicerrectores, genera desencuentros que afectan la marcha administrativa integral. Atenta contra el liderazgo eficaz.

5. Ausencia de coordinación inter Facultades, bajo el concepto de la autonomía académica, económica, normativa y administrativa.

6. Multiplicidad de organismos que realizan una misma función.

Decanos: 20 decanatos de Facultades.

Directores: 20 Institutos de Investigación y 20 Unidades de Posgrado.

Jefes: 20 Unidades de Personal; 20 Unidades de Trámite Documentario; 20 Unidades de Bienestar; 20 Unidades de Orientación Estudiantil.

Secretarias: 20 Secretarias de Decano; 20 Secretarias en los Institutos de Investigación; 20 Secretarias en las Unidades de Posgrado.

Lo que demuestra falta de racionalización y eficiencia administrativa.

7. Escasa comunicación horizontal inter facultades para trabajo en equipo por áreas afines. Prima la información vertical y con dificultades.

8. Hay competencia académico-económico-administrativa inter facultades, cuando esta debería ser corporativa ante las universidades nacionales e internacionales. Por tanto, el concepto de "competencia” está distorsionado.

9. Se toman decisiones pensando en la "Facultad" y no en nuestra "Universidad". Lo que genera un desperdicio de los escasos recursos disponibles, tanto monetarios como de infraestructura.

10. Como los sueldos son bajos todos los docentes aspiran a cargos académico-administrativos, olvidando el objetivo principal que les compete: 
docencia e investigación. Así, se ha generado una institución de competencia política electoral para satisfacer apetitos personales que se dedica a promover la producción científica.

11. En este tipo de organización el poder del voto está sobre el talento y ha convertido a la organización universitaria en distritos electorales de docentes y estudiantes que no contribuyen a la toma de decisiones estratégicas de desarrollo a largo plazo.

12. La organización por facultades tradicionales, rígida y encapsulada, no admite alternativas de interdisciplinariedad, donde los profesores deberían estar asignados a la universidad y no a la facultad.

13. Hay modelos universitarios de promover el desarrollo y otros, el subdesarrollo. La experiencia nos demuestra que el modelo que fenece tiende a la afirmación del subdesarrollo.

\section{LA REINGENIERÍA QUE NECESITA SAN MARCOS}

La propuesta específica es convertir las 20 facultades vigentes e integrarlas solamente a cinco (5) por especialización: Ciencias Básicas; Ciencias de la Salud; Ingenierías; Económico-Empresariales y Humanidades ${ }^{1}$. Una nueva estructura simplificada que facilitará la eficacia, la eficiencia y la efectividad en la toma de decisiones económico-administrativas.

\section{LAS NUEVAS FACULTADES INTEGRADAS}

En los cinco (5) organigramas que estamos proponiendo (ver ANEXOS cuadros $\mathrm{N}^{\circ} \mathrm{s} 05,06,07,08$, y09), todas las facultades tienen la misma estructura:

a) Consejo de Facultad.

b) Decanato.

c) Escuelas Académico Profesionales.

d) Centros de Producción.

e) Institutos de Investigación.

f) Unidades de Posgrado.

g) Responsabilidad Social Universitaria (nueva área que exige la norma vigente).

h) Coordinación Académica. i) Imagen Institucional.

j) Secretaría de Administración.

k) Secretaría Académica.

\section{El Consejo de Facultad}

Según el artículo 670 de la Ley, este órgano de gobierno está integrado por:

“67.1.1. El Decano, que lo preside”.

“67.1.2. Los representantes de los docentes. Su número está establecido en el Estatuto de cada universidad. Proponemos mantener la misma proporción que exige el artículo $56^{\circ}$ para la formación de la Asamblea Universitaria: 50\% de Profesores Principales, 30\% de Profesores Asociados y 20\% de Profesores Auxiliares. Es decir, 05 Principales; 03 Asociados y 02 Auxiliares de los profesores que obtengan el mayor número de votos en las elecciones universales".

“67.1.3 Los representantes de los estudiantes regulares, que constituyen un tercio del total de integrantes del Consejo, según corresponda. Es decir, 04 alumnos (33\% de 11 Profesores incluido el Decano)".

Una alternativa de solución para la Asamblea Estatutaria sería nombrar Comisiones de Coordinación en:

Escuelas Académico Profesionales: Representado por los Directores de las Escuelas Académico Profesionales, por ejemplo: Ciencias Biológicas, Ciencias Físicas, Ciencias Matemáticas, y así sucesivamente, reunidos para discutir cómo facilitar el desarrollo de las actividades a futuro, actualización de reglamentos y organigrama del área.

Centros de Producción: Representado por sus Directores y/o encargados de los Centros de Producción, por ejemplo: Farmacia y Bioquímica, Medicina Humana, Medicina Veterinaria, Odontología, Psicología, reunidos para discutir cómo facilitar el desarrollo de las actividades a futuro, actualización de reglamentos y organigrama del área.

Institutos de Investigación: Representado por sus Directores y/o encargados de los Institutos de Investigación, por ejemplo de: Ingeniería Geológica, Minera, Metalúrgica, Geografía; Química e

1 Ver detalle en las páginas anteriores: Estructura Organizacional de los Institutos de Investigación.

14/ QVIPURAMAYOC | Vol. 22(42) 2014 
Ingeniería Química; Ingeniería Electrónica y Eléctrica; Ingeniería Industrial e Ingeniería de Sistemas e Informática, reunidos para discutir cómo facilitar el desarrollo de las actividades a futuro, actualización de reglamentos y organigrama del área.

Unidades de Posgrado: Representado por sus Directores y/o funcionarios de las Unidades de Posgrado, por ejemplo: Ingeniería Geológica, Minera, Metalúrgica, Geográfica; Química e Ingeniería Química; Ingeniería Electrónica y Electrica; Ingeniería Industrial e Ingeniería de Sistemas e Informática, reunidos para discutir cómo facilitar el desarrollo de las actividades a futuro, actualización de reglamentos y organigrama del área.

Responsabilidad Social Universitaria: Integrado por sus Directores y/o representantes de las oficinas a fenecer de los Centros de Extensión y Proyección Social de cada área, reunidos para discutir cómo implementar las nuevas actividades que exige la Ley vigente de Universidades.

Coordinación Académica: Integrado por sus Directores y/o representantes de Coordinación Académica, por ejemplo: Ciencias Administrativas, Ciencias Contables y Ciencias Económicas, reunidos para discutir cómo facilitar el desarrollo de las actividades a futuro, actualización de reglamentos y organigrama del área.

Imagen Institucional: Integrado por los funcionarios y/o personas que desarrollaban estas actividades en las facultades tradicionales, por ejemplo: Derecho y Ciencia Política, Ciencias Sociales, Educación, Letras y Ciencias Humanas, reunidos para discutir cómo facilitar el desarrollo de las actividades a futuro, actualización de reglamentos y organigrama del área.

Secretaría de Administración: Integrado por sus Directores y/o representantes del área, por ejemplo: Ciencias Administrativas, Ciencias Contables y Ciencias Económicas, reunidos para discutir cómo facilitar el desarrollo de las actividades a futuro, actualización de reglamentos y organigrama del sector.

Estas comisiones de trabajo se replicarían en cada una de las cinco (5) Facultades propuestas y tendrían 55 días calendarios según la Ley (Disposición complementaria transitoria). En realidad, no se necesita más de 30 días, y remunerada en función de objetivos y metas alcanzadas.

\section{El Nuevo Consejo Superior de Investiga- CIONES}

Para eliminar los "cuellos de botella" que se presentan en la actual forma de asignar los Proyectos de Investigación, sugerimos a la Asamblea Estatutaria, al momento de actualizar el Reglamento de las Investigaciones Multidisciplinarias, realizar el siguiente procedimiento:

1)Desarrollar los Fondos Concursales a la totalidad de Proyectos (En la actualidad 25, si fuera el caso del área Económico-Empresariales), no por facultades como se desarrolla ahora, sino por áreas.

2) Nombrar un Equipo de seis (6) Investigadores procedentes de los tres (03) Institutos de Investigación elegidos de una terna propuesta por cada organismo para formar el Jurado Calificador, y elegidos por la Dirección del Consejo Superior de Investigaciones. De esta manera la calificación sería más transparente.

3) Si se tratara del área Económico-Empresarial, por ejemplo, los 25 mejores proyectos serán calificados como ganadores, beneficiando a los trabajos mejor sustentados sin tener en cuenta la facultad procedente; generando internamente una sana competencia y promoviendo así, a diferentes facultades, eliminando los vicios de asignación de Proyectos por influencia política de grupo e integrando paulatinamente a nivel superior a los investigadores para la formulación de Proyectos Interdisciplinarios.

4)Este procedimiento se replicaría en las demás áreas o futuras facultades.

5)El centro de operaciones de esta área sería el Consejo Superior de Investigaciones, que promueve anualmente fondos concursales en forma coordinada con los Institutos.

Esta estructura organizacional no promueve la investigación multidisciplinaria sino unidisciplinaria, relacionado con la facultad a que corresponde. 
6) La medida correctiva sería promover la investigación multidisciplinaria, teniendo como base a las Ciencias Empresariales.

7) Integrar por áreas los Institutos de Investigación. Ejemplo: En lugar de tener 20 Institutos (uno por cada facultad), en el área Económico-Empresariales solo habría un instituto, así se reduciría a 5, uno por área.

8)Integrar las Revistas de Investigación. Ejemplo: En el área Económico-Empresariales solo sería una revista en lugar de 3. A nivel de universidad, en lugar de tener 20, se reduciría a 5: uno por cada área. Adicionar las revistas antiguas que tienen su prestigio ganado en la Academia, lo que harían un total de 8 , además de la reducción de costos.

9) ¿Cómo hacerlo? Formando las comisiones para cada área:

1) Institutos integrados.

2) Revistas de investigación integrados.

3) Preparación del nuevo Estatuto repartido entre las 12 Comisiones según cronograma de Actividades propuesto.

(Véase en páginas posteriores)

\section{BENEFICIOS E INCONVENIENTES DE LA PROPUESTA}

\section{Beneficios}

1. Eliminación de barreras físicas, actualmente existentes entre las diferentes Facultades y Escuelas.

2. Mayor coordinación en cada área entre directivos, personal docente y administrativo.

3. Simplificación del control administrativo. En lugar de tener 20 Facultades, se reduciría a 5.

4. Mejora en la coordinación entre las Unidades de Posgrado: Contenido; denominación de los cursos; uniformidad del cobro de pensiones de enseñanza y estandarización de cursos a nivel propedéutico.

5. Revisión de los contenidos de cada especialidad de acuerdo a sus campos privativos: Administradores, Contadores, Economistas.

6. Centralización de los Institutos de Investigación, ahorrando ambientes y destinándolos a otras prio- ridades; permitiendo la investigación por grupos polivalentes, y centralizando las publicaciones en menor cantidad de revistas pero con mayor calidad y continuidad.

7. Mejora en la evaluación y actualización de objetivos de Maestrías y Doctorados.

8. Creación de una nueva cultura en la admisión de postulantes, orientándolos a programas académico-profesionales en las áreas que el país necesita desarrollar.

9. Modernización del Reglamento Interno del Otorgamiento de Grados, de acuerdo a las exigencias del mercado actual, exigiendo obligatoriamente la Tesis de Grado para obteenr el Bachillerato.

10. Promoción de los sectores económicos que generan empleo en coordinación con los gremios institucionales.

11. Alianzas estratégicas con asociaciones empresariales, promoviendo acuerdos de cooperación en sectores de mutuo beneficio.

12. Mejoras de las ventajas competitivas de las empresas relacionadas a proyectos contratados con la universidad.

13. Capacidad de respuesta de la universidad frente a las necesidades del entorno, porque facilitará definir políticas claras de franco apoyo a la industrialización del país.

14. Creación de una cultura prospectiva en las autoridades, a pensar en el futuro, sin dejar de lado el análisis del presente.

15. Simplificación en la toma de decisiones al eliminar Decanaturas, Direcciones administrativas y Direcciones Académicas que se repetían en 15 facultades que desaparecerían.

16. Optimización de la eficiencia competitiva, el control de costos y la acreditación que en el futuro será una norma de exigencia de calidad de los servicios ofrecidos por la universidad.

17. Buenas prácticas de la transparencia, en eficiente uso de los recursos disponibles y en la toma de decisiones más adecuadas. 


\section{Inconvenientes}

1. Riesgo de politización del debate técnico.

2. Necesidad de inversión en un software integral para el control de las operaciones contable-administrativas.

3. Costo de liquidación del personal excedente.

4. Resistencia al cambio del personal involucrado.

\section{FUNCIONES DE LA NUEVA ASAMBLEA ESTATUTARIA}

Según los dispositivos de la Ley, Primera Disposición Complementaria, la Asamblea Estatutaria redacta y aprueba el Estatuto de la Universidad, en un plazo de cincuenta y cinco (55), días calendario y está conformada por 36 miembros:

- 12 Profesores Principales.

- 08 Profesores Asociados

- 04 Profesores Auxiliares

- 12 Estudiantes.

Precisa del apoyo de la planificación administrativa para desarrollar con la mayor eficiencia el empleo de su tiempo.En teoría, podría dividirse en 12 comisiones integradas por tres miembros $(12 * 3=36)$, y presidido por un Profesor Principal y completada por un alumno del Tercio. Los Profesores Principales participarán en las Comisiones más importantes y se complementará con la presencia de los Profesores Auxiliares. 


\section{CRONOGRAMA DE ACTIVIDADES}

\begin{tabular}{|c|c|c|c|c|c|c|c|c|}
\hline \multirow{2}{*}{ ACTIVIDADES } & \multicolumn{8}{|c|}{ SEMANAS } \\
\hline & 1 & 2 & 3 & 4 & 5 & 6 & 7 & 8 \\
\hline $\begin{array}{l}\text { Primera Reunión. Comisión Estatutaria define la estructura organi- } \\
\text { zacional. Mantiene la actual o crea una nueva. Asumimos que optará } \\
\text { por desarrollar una nueva. }\end{array}$ & $\mathrm{xx}$ & & & & & & & \\
\hline $\begin{array}{l}\text { Comisión 1. Desarrollar el borrador del Estatuto: Asamblea } \\
\text { Universitaria, Consejo Universitario y Rectorado. }\end{array}$ & $\mathrm{xx}$ & $\mathrm{xx}$ & $\mathrm{xx}$ & $\mathrm{xx}$ & & & & \\
\hline $\begin{array}{l}\text { Comisión 2. Desarrollar el borrador del Estatuto: Secretaría General, } \\
\text { Oficina de Relaciones Interinstitucionales, Museos y Oficina } \\
\text { General de Asesoría Legal. }\end{array}$ & $\mathrm{xx}$ & $\mathrm{xx}$ & $\mathrm{xx}$ & $\mathrm{xx}$ & & & & \\
\hline $\begin{array}{l}\text { Comisión 3. Desarrollar el borrador del Estatuto: Dirección General } \\
\text { de Administración y Vicerrectorado Académico. }\end{array}$ & $\mathrm{xx}$ & $\mathrm{xx}$ & $\mathrm{xx}$ & $\mathrm{xx}$ & & & & \\
\hline $\begin{array}{l}\text { Comisión 4. Desarrollar el borrador del Estatuto: Vicerrectorado de } \\
\text { Investigación, Institutos de Investigación de la Universidad e integra- } \\
\text { ción de revistas de Investigación. }\end{array}$ & $\mathrm{xx}$ & $\mathrm{xx}$ & $\mathrm{xx}$ & $\mathrm{xx}$ & & & & \\
\hline $\begin{array}{l}\text { Comisión 5. Desarrollar el borrador del Estatuto: Facultad de } \\
\text { Ciencias Básicas. }\end{array}$ & $x x$ & $\mathrm{xx}$ & $\mathrm{xx}$ & $x x$ & & & & \\
\hline $\begin{array}{l}\text { Comisión 6. Desarrollar el borrador del Estatuto: Facultad de } \\
\text { Ciencias de la Salud. }\end{array}$ & $\mathrm{xx}$ & $\mathrm{xx}$ & $\mathrm{xx}$ & $x x$ & & & & \\
\hline $\begin{array}{l}\text { Comisión 7. Desarrollar el borrador del Estatuto: Facultad de } \\
\text { Ingenierías. }\end{array}$ & $\mathrm{xx}$ & $\mathrm{xx}$ & $\mathrm{xx}$ & $\mathrm{xx}$ & & & & \\
\hline $\begin{array}{l}\text { Comisión 8. Desarrollar el borrador del Estatuto: Facultad de } \\
\text { Económico-Empresariales. }\end{array}$ & $\mathrm{xx}$ & $\mathrm{xx}$ & $\mathrm{xx}$ & $\mathrm{xx}$ & & & & \\
\hline $\begin{array}{l}\text { Comisión 9. Desarrollar el borrador del Estatuto: Facultad de } \\
\text { Humanidades. }\end{array}$ & $\mathrm{xx}$ & $\mathrm{xx}$ & $\mathrm{xx}$ & $\mathrm{xx}$ & & & & \\
\hline $\begin{array}{l}\text { Comisión 10. Desarrollar el borrador del Estatuto: Escuela de } \\
\text { Posgrado y las Unidades de Posgrado de la Universidad. }\end{array}$ & $\mathrm{xx}$ & $\mathrm{xx}$ & $\mathrm{xx}$ & $\mathrm{xx}$ & & & & \\
\hline $\begin{array}{l}\text { Comisión 11. Desarrollar el borrador del Estatuto: Centros de } \\
\text { Producción de la Universidad }\end{array}$ & $\mathrm{xx}$ & $\mathrm{xx}$ & $\mathrm{xx}$ & $\mathrm{xx}$ & & & & \\
\hline $\begin{array}{l}\text { Comisión 12. Desarrollar el borrador del Reglamento de } \\
\text { Organización y Funciones de la Universidad }\end{array}$ & $x x$ & $\mathrm{xx}$ & $\mathrm{xx}$ & $x x$ & & & & \\
\hline $\begin{array}{l}\text { Asamblea General de la Comisión Estatutaria para aprobar Nuevo } \\
\text { Estatuto de la Universidad }\end{array}$ & & & & & & $\mathrm{xx}$ & & \\
\hline $\begin{array}{l}\text { Asamblea General de la Comisión Estatutaria para aprobar Nuevo } \\
\text { Reglamento de Organización y Funciones de la Universidad }\end{array}$ & & & & & & & $x x$ & $\mathrm{xx}$ \\
\hline $\begin{array}{l}\text { Recepción de Consultas de los Grupos de Interés sobre borrador del } \\
\text { Estatuto y Reglamento. }\end{array}$ & & & & & & & $\mathrm{xx}$ & \\
\hline Clausura funciones de Comisión Estatutaria & & & & & & & & $\mathrm{xx}$ \\
\hline
\end{tabular}

Fuente: Nuevo Organigrama Estructural propuesto (Ver ANEXOS Cuadro № 01) Material disponible para las Comisiones:

a) Copia de Nueva Ley Universitaria; b) Copia del anterior Estatuto de la Universidad; c) Copia de los organigramas propuestos. 


\section{CONCLUSIONES}

1. La investigación multidisciplinaria es una necesidad fundamental para que nuestra universidad mejore su posición en el contexto internacional. Alcanzar esta meta sólo será posible integrando las diferentes 20 Facultades a solo cinco (5): Ciencias Básicas, Ciencias de la Salud, Ingenierías, Económico-Empresariales y Humanidades, coordinado con un Reglamento Interno de Investigaciones actualizado y proyectado al futuro. Asimismo, agregar la Escuela de Posgrado.

2. Transparencia, porque con la nueva Ley, el Rector tiene la obligación de presentar a la Asamblea Universitaria el informe semestral de gestión, informe de rendición de cuentas del presupuesto anual y la Memoria Anual. Se plantea la reducción de 15 posiciones en 5 áreas que a un promedio de $\mathrm{S} / .10,000$ mensuales, por doce meses, genera una reducción de gastos en beneficio de la investigación de 9 millones de soles como mínimo por año.

3. Simplificación administrativa, porque la integración de los diferentes sectores de la universidad más antigua de América, facilitará el manejo de la base de datos con el apoyo de las Tecnologías de Información y Comunicación dirigidas desde una nueva Oficina General de Administración de la Red Telemática, dependiente directamente del Director General de Administración.

\section{REFERENCIA BIBLIOGRÁFICA}

1. ARRARTE, R. (2005). La Cooperación Universidad-Empresa-Estado en el Perú (Caso UNMSM 1997-2002). Tesis de Doctorado. UNMSM. Lima. Perú.

\section{ANEXOS}

Cuadro No 01: Organigrama estructural UNMSM 2014

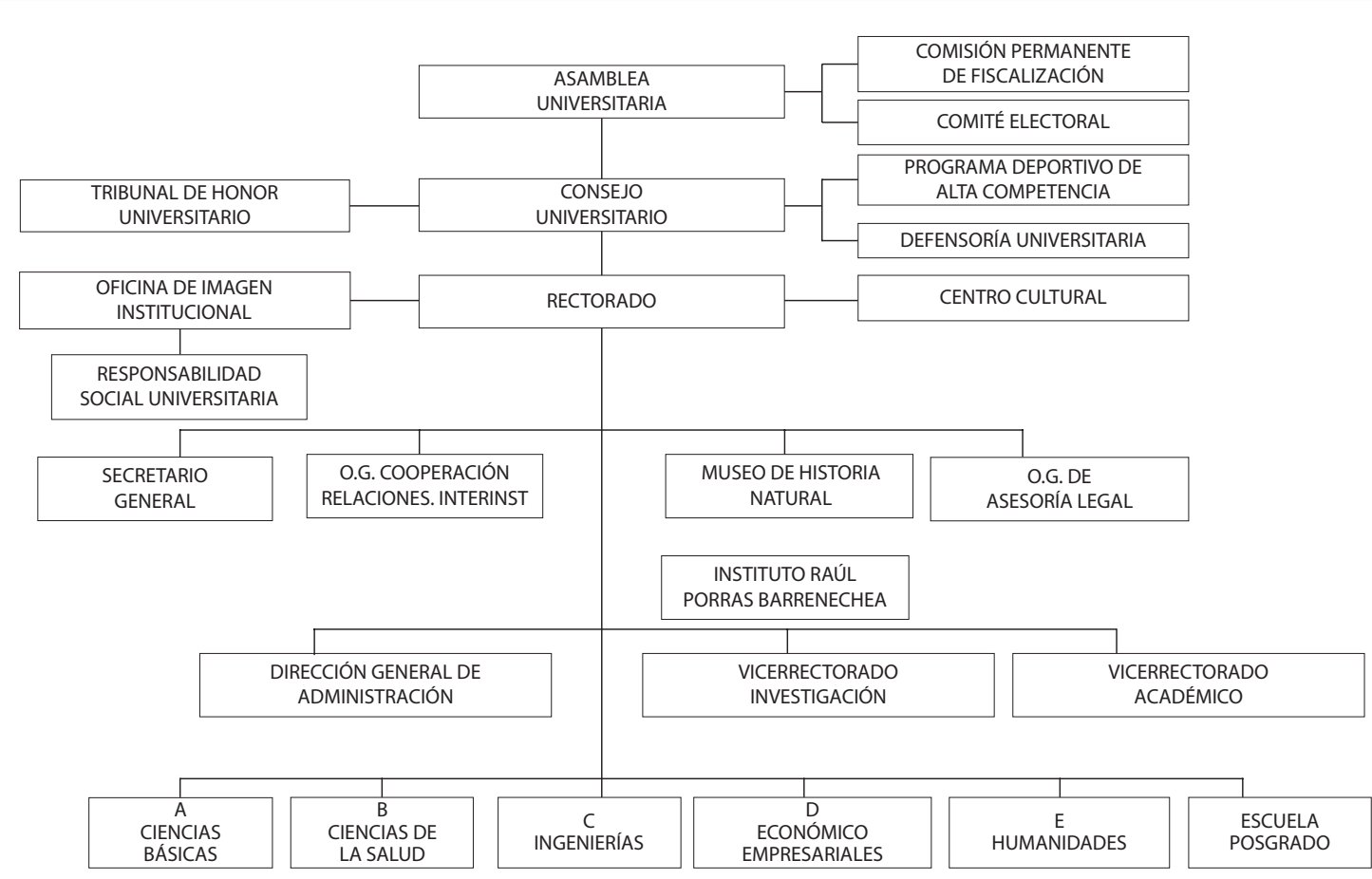

ÁREAS COORDINACIÓN

A = Ciencias Biológicas, Ciencias Físicas, Ciencias Matemáticas

B = Bioquímica, Farmacia, Medicina Humana, Medicina Veterinaria, Odontología, Psicología

C = Geografía, Geología, Ing. de Sistemas, Ing. Electrónica, Ing. Industrial, Ing. Química, Química

$C=$ Geografia, Geologia, Ing. de Sistemas, Ing. Electrónica, Ing. Industria
$D=$ Ciencias Administrativas, Ciencias Contables, Ciencias Económicas

$\mathrm{E}=$ Ciencias Sociales, Derecho y Ciencia Política, Educación, Letras y Ciencias Humanas 
Cuadro No 02: Dirección General de Administración

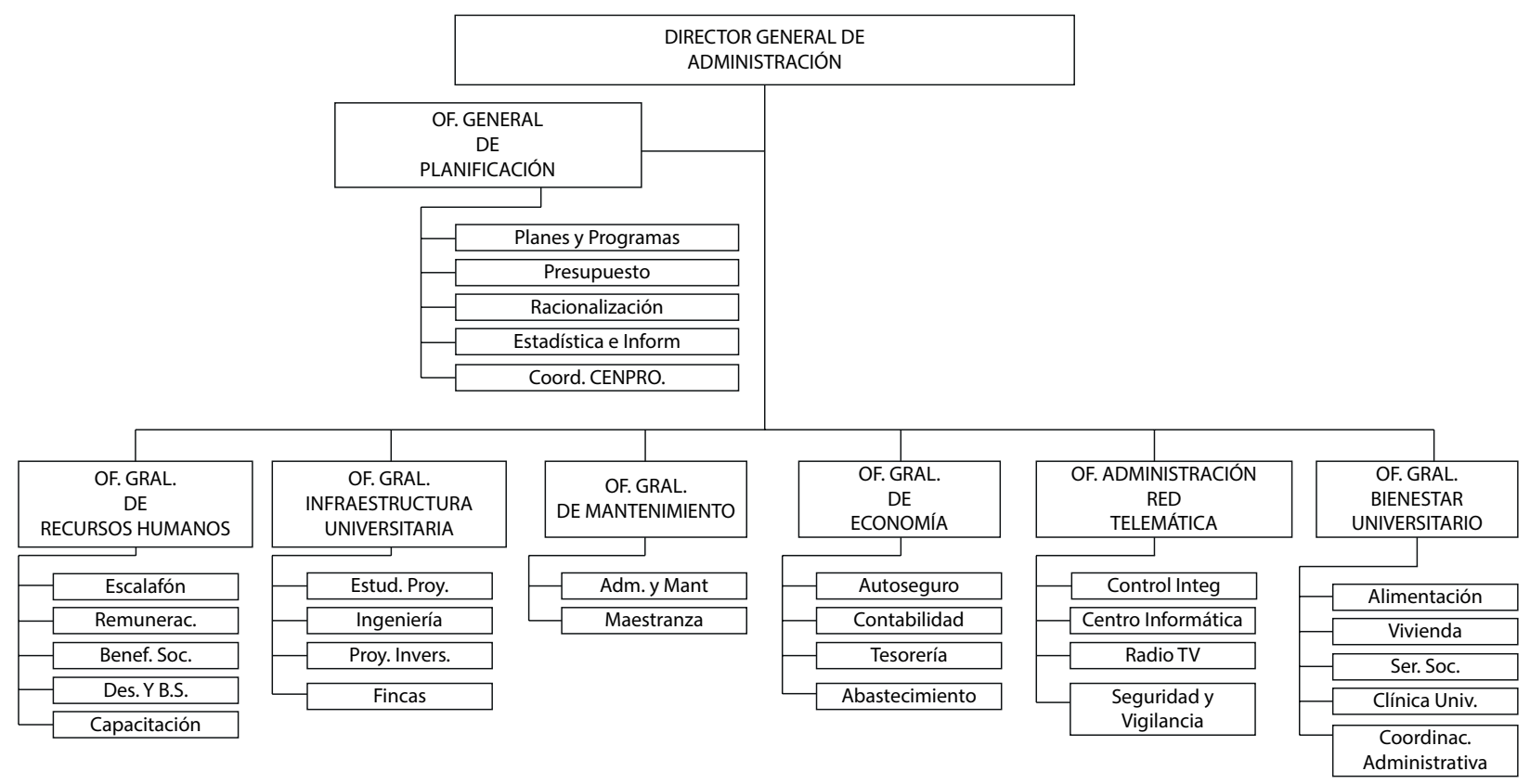

Cuadro No 03: Vicerrectorado Académico

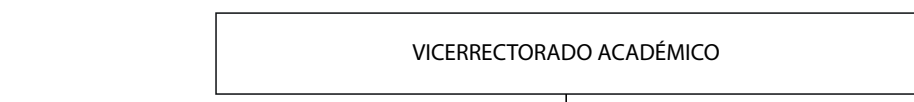


Cuadro No 04: Vicerrectorado de Investigaciones

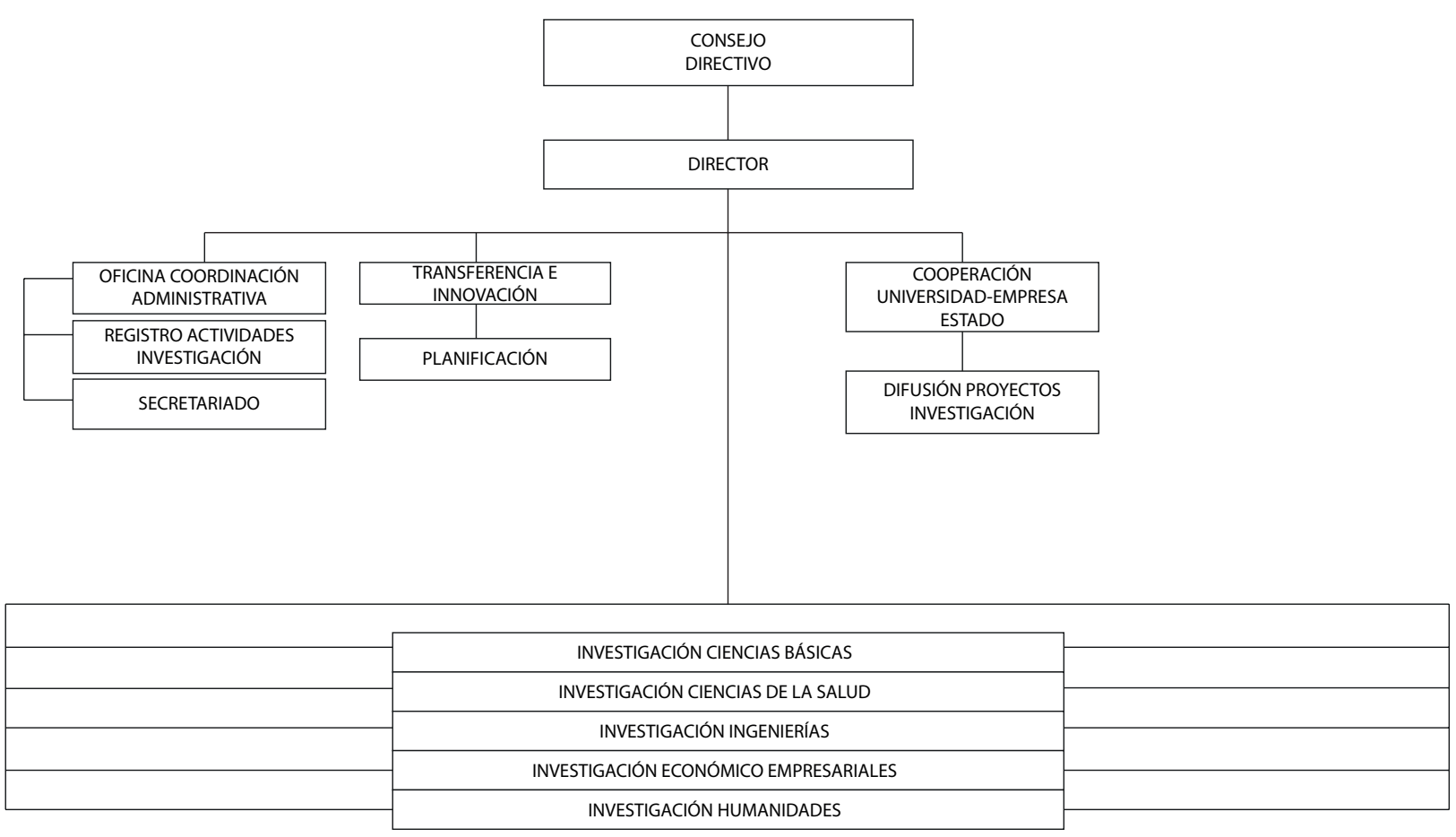

\section{VICERRECTORADO INVESTIGACIONES}

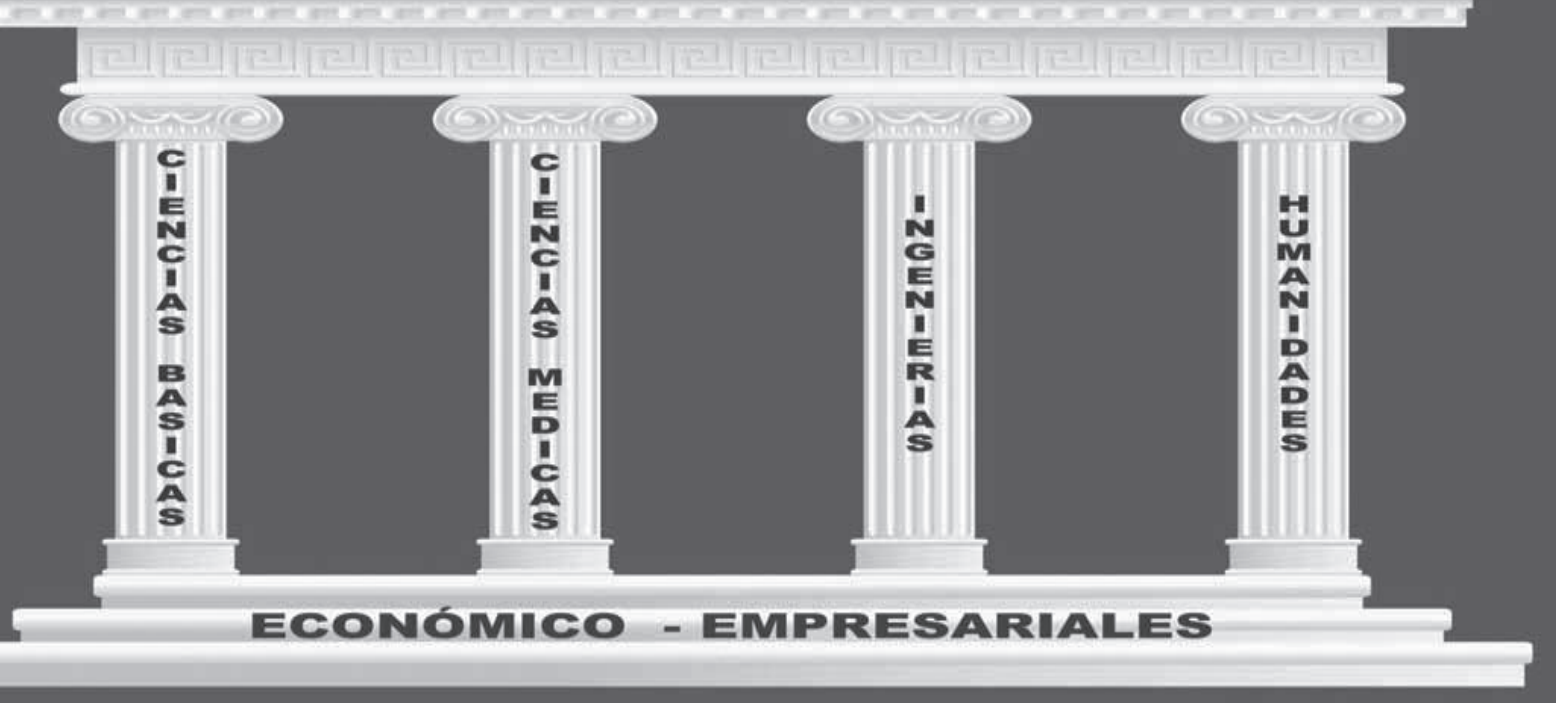

Vol. 22(42) 2014 | QUIPURAMAYOC / 21 
Cuadro No 05: Facultad de Ciencias Básicas

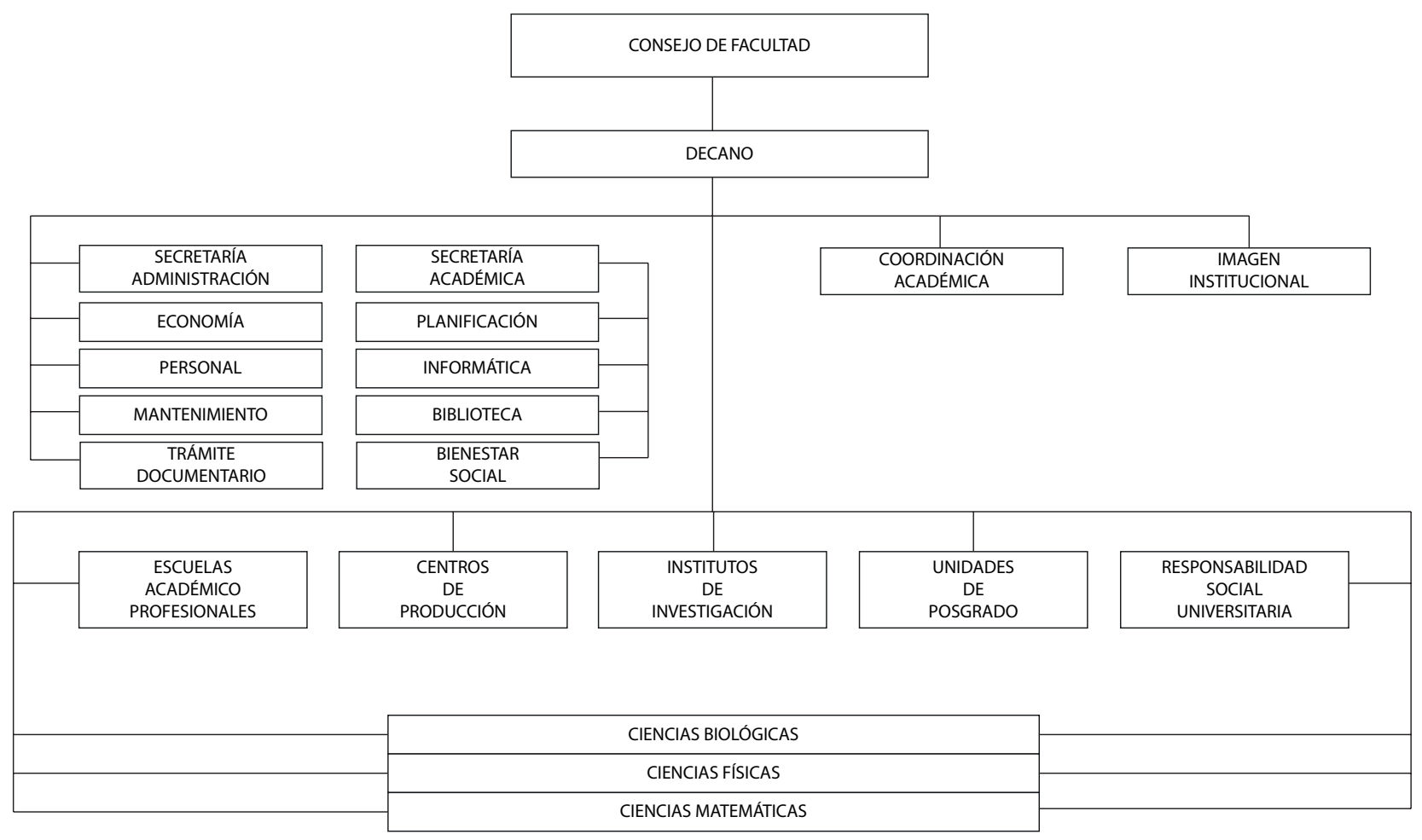

Cuadro No 06: Facultad de Ciencias de la Salud

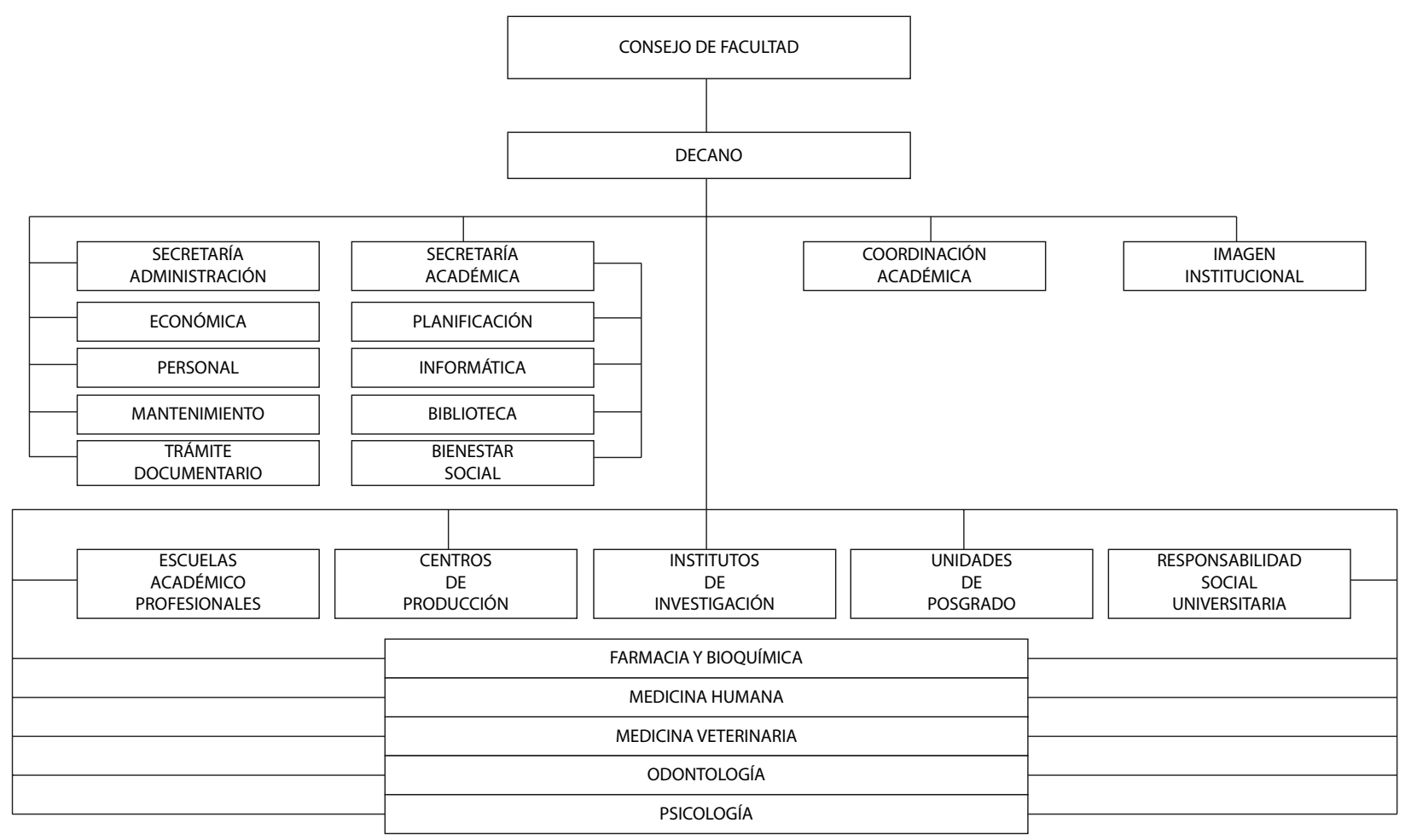


Cuadro No 07: Facultad de Ingenierías

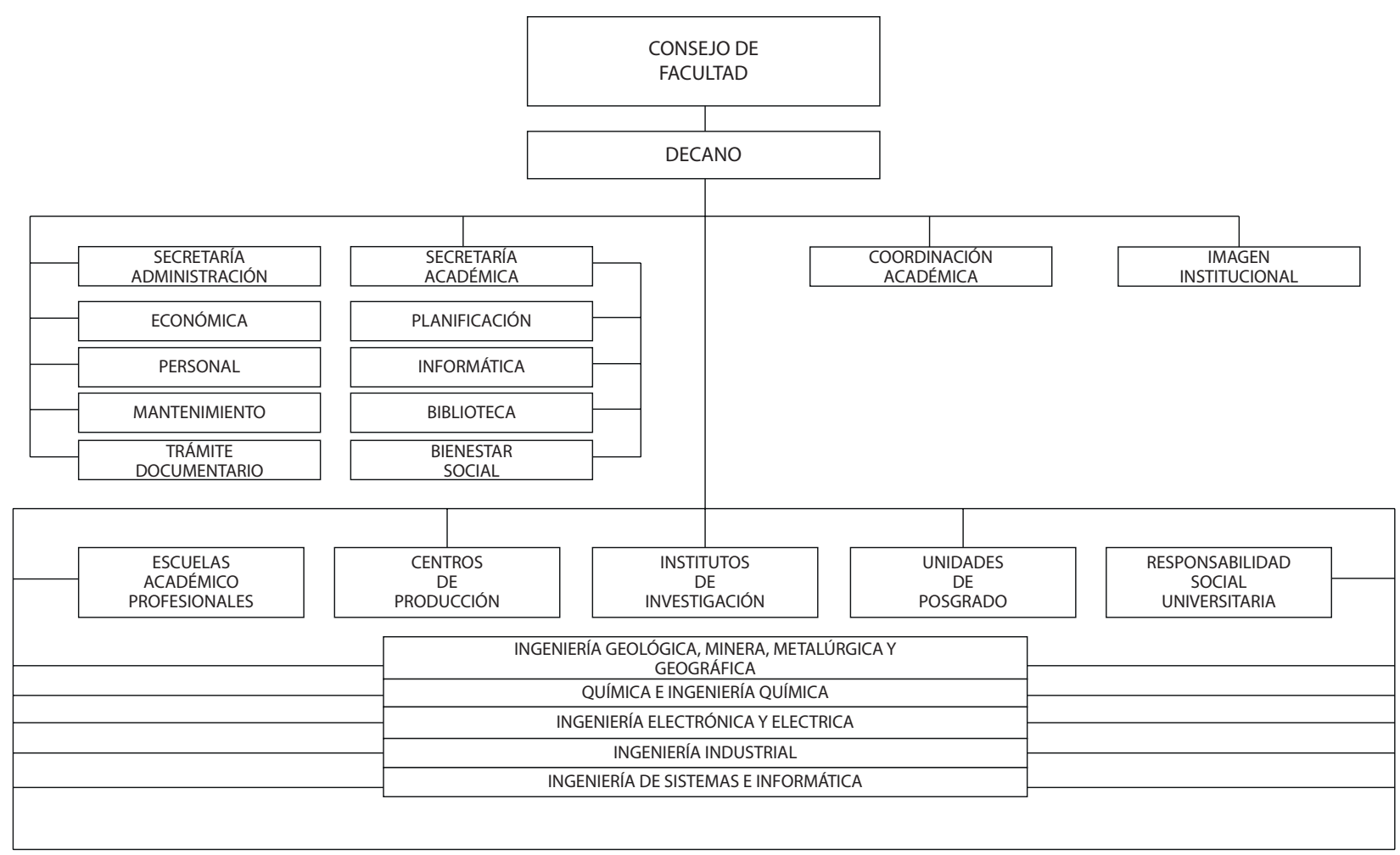

Cuadro No 08: Facultad Ciencias Económico - Empresariales

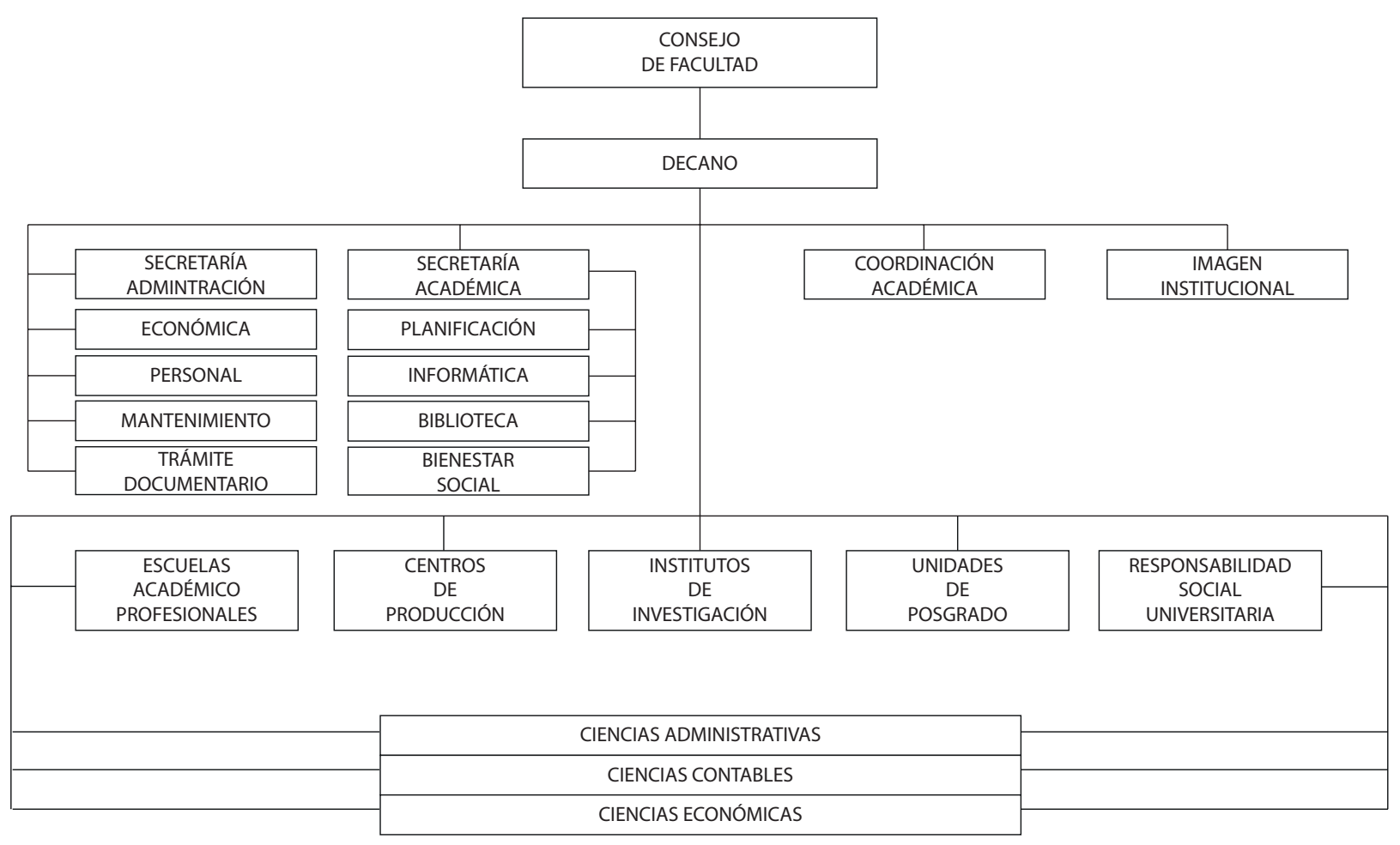

Vol. 22(42) 2014 | QUIPURAMAVOC / 23 
Cuadro No 09: Facultad de Humanidades

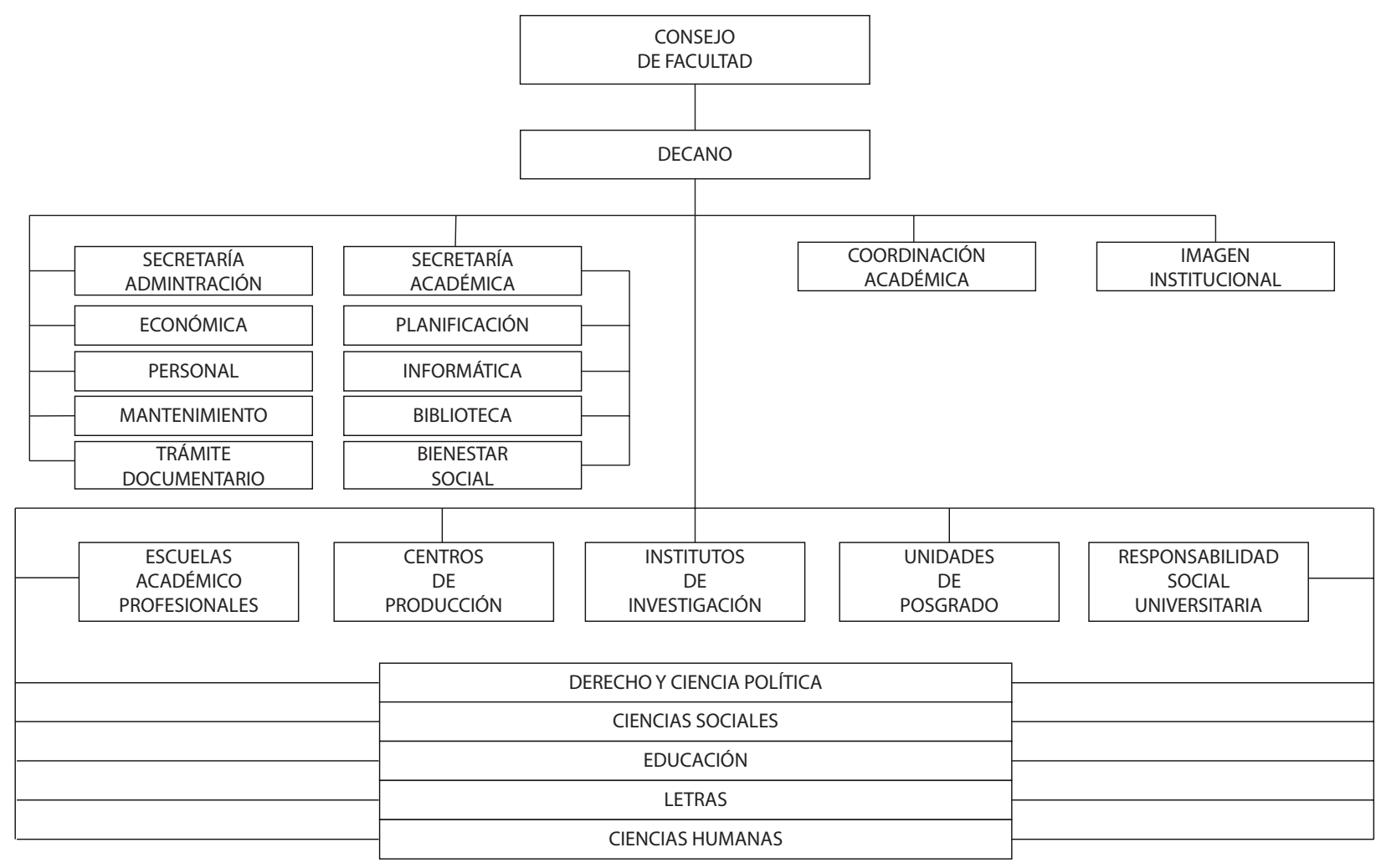

Cuadro No 10: Escuelas de Posgrado

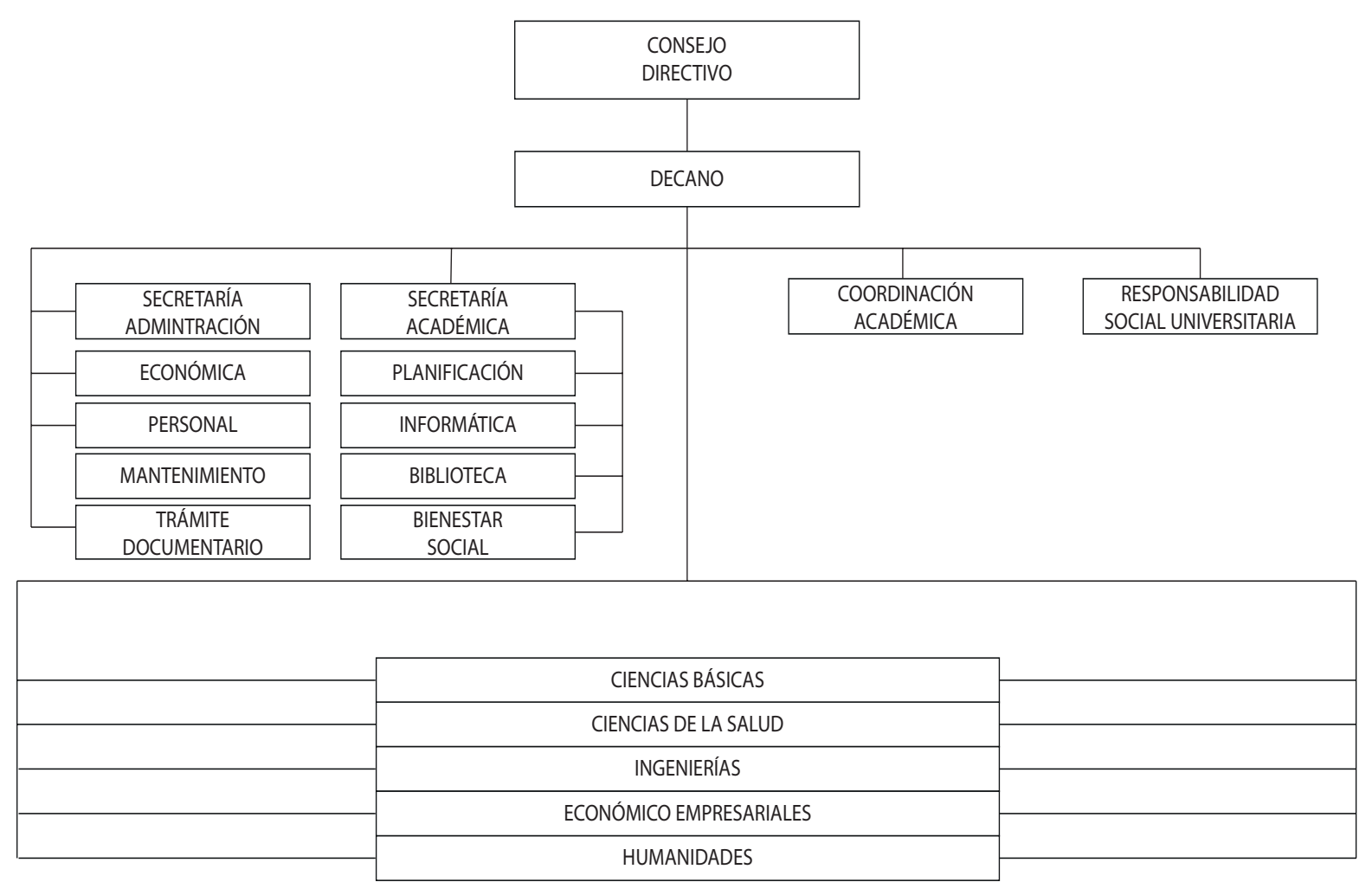

24/ Quipuramavoc|Vol. 22(42) 2014 